\title{
Atribución de factores de riesgo de suicidio en niños y adolescentes en la comunidad inmigrante latina: una muestra del sur de California
}

\author{
Noelia NAVARRO*1, Luis GARCÍA** y Adolfo J. CANGAS* \\ (*) Universidad de Almería, España (**) Pacific Clinics, CA, USA
}

(Received on August 8, 2016; Accepted on October 12, 2016)

\begin{abstract}
In the last years an alarming increase has taken place in the number of suicides in children and teenagers, being a daily reality in the panorama of the USA, especially in the case of Latino immigrants. In the current study, a survey about the perceived risk factors for suicide was established between risk population. The sample was composed of 593 children and adolescents aged between 11 and 18 years, most Latino ethnicity, belonging to "Latina Youth Program" (LYP), a program of suicide prevention in risk population, developed by Pacifics Clinics, an organization that operates in Los Angeles, California. They interviewed the participants about the factors they considered risk for suicidal behavior. Participants were randomly selected in 2003, 2005 and 2008. The factors most commonly identified risk were the regulation of emotions (96.57\%), poor family communication, poor school performance, the influence of the peer group (89.67\%, 82. 73\% and $41.57 \%$ respectively). The found trend is towards an increase in the perception of risk factors detected in any case up to ten risk factors for suicide. The higher quantity of variables impede the precise determination of the (s) reason (s) that might end in this fatidical conclusion, being the most common a panorama in which converge multitude of variables, crucial to settling objectives in interventions.
\end{abstract}

Keywords: Suicide, latins, risk factor.

Attribution of risk factors for suicide in children and adolescents in the Latino immigrant community: a sample of Southern California

RESUMEN: En los últimos años se ha producido un incremento alarmante en el número de suicidios en niños y adolescentes, siendo una realidad cotidiana en el panorama de los EEUU, especialmente en el caso de inmigrantes latinos. En el presente estudio, se estableció un sondeo en población de riesgo acerca de los factores de riesgo de suicidio percibidos. La muestra quedó conformada por 593 niños y adolescentes de edades comprendidas entre los 11 y los 18 años, la mayoría de etnia latina, pertenecientes a "Latina Youth Program" (LYP), un programa de prevención del suicidio en población de riesgo, desarrollado por Pacifics Clinics, una organización que desarrolla su actividad en Los Ángeles, California. Se entrevistó a los participantes acerca de los factores que ellos consideraban de riesgo para la conducta suicida. Los participantes fueron seleccionados aleatoriamente en los años 2003, 2005 y 2008. Los factores de riesgo más comúnmente señalados fueron la regulación de emociones (96.57\%), la pobre comunicación familiar, bajo rendimiento escolar, la influencia del grupo de iguales (89.67\%, 82. 73\% y 41.57\% respectivamente). La tendencia encontrada apunta hacia un aumento en la percepción de factores de riesgo, detectándose en cualquier caso hasta diez factores de 
riesgo de suicidio. La cantidad de variables puestas en juego complejizan la determinación precisa de la(s) causa(s) que podrían desembocar en este fatídico desenlace, siendo lo más común un panorama en el que confluyen multitud de variables, cruciales para asentar objetivos en las intervenciones.

Palabras clave: Suicidio, latinos, factores de riesgo.

${ }^{1}$ Correspondence: Noelia Navarro. Universidad de Almería, edificio C, Crta Sacramento, s/n. La Cañada de San Urbano, 04120 Almería. E-mail: nng777@ual.es.

El suicidio, un fenómeno multifactorial, constituye una de las principales problemáticas inherentes a la población de USA, agudizándose la problemática al pormenorizar en las particularidades circundantes a la infancia, adolescencia y juventud. El suicidio se define como un acto deliberadamente iniciado y realizado por una persona en pleno conocimiento o expectativa de su desenlace fatal (Organización Mundial de la Salud; OMS, 2014). No estamos, por tanto, ante una cuestión banal, sino que se trata de un problema de salud pública, estando considerada la tasa de suicidio como un indicador de la salud mental de la población de un país.

En líneas generales, dadas las estadísticas disponibles podría concluirse que la permanencia y extensión del suicidio es una práctica generalizada en todas las sociedades, siendo un fenómeno complejo y multicausal, de necesidad acuciante dada la repercusión en el entorno social más próximo de la víctima y los años de vida perdidos.

En lo concerniente a los EEUU, aunque durante los últimos veinte años, las tasas de suicidio en la población general, parecen haberse estabilizado, ésta continúa siendo una de las principales causas de mortalidad en todos los rangos de edad. De hecho, los datos más recientes disponibles apuntan a un incremento próximo al $24 \%$ en la tasa de suicidio en los EEUU en el período comprendido entre 1999 y 2004, siendo esta cifra suficiente para situar la causa suicida como la décima en lo que a mortalidad se refiere (ver tabla 1, Centros para el control y la prevención de enfermedades, 2016), invirtiendo en cualquier caso la tendencia imperante durante la última década y media.

Tabla 1. Diez causas principales de mortalidad en jóvenes estadounidenses en 2013 (tomado de Jiaquan, Sherry, Kenneth y Brigham, 2016)

\begin{tabular}{|c|c|c|c|}
\hline \multicolumn{4}{|c|}{ Rango de edad 1-85 años } \\
\hline $\mathbf{1}$ & Enfermedad cardíaca & $\mathbf{6}$ & $\begin{array}{c}\text { Alzheimer } \\
84.767\end{array}$ \\
\hline $\mathbf{2}$ & Neoplasma maligno & $\mathbf{7}$ & $\begin{array}{c}\text { Diabetes Mellitus } \\
75.578\end{array}$ \\
\hline $\mathbf{3}$ & Enfermedad respiratoria crónica & $\mathbf{8}$ & Gripe y Neumonía \\
& 149.205 & & 56.979 \\
\hline $\mathbf{4}$ & Accidente no intencionado & $\mathbf{9}$ & Nefritis \\
& 130.557 & & 47.112 \\
\hline $\mathbf{5}$ & Enfermedad cerebrovascular & $\mathbf{1 0}$ & Suicidio \\
& 128.978 & & $\mathbf{4 1 . 1 4 9}$ \\
\hline
\end{tabular}

Haciendo alusión al caso de jóvenes, el suicidio es la segunda causa principal de muerte a nivel mundial en el grupo de 15 a 29 años (Organización Mundial de la Salud, OMS, 2014). En EEUU, se registran más muertes por suicidio que por accidentes de tráfico. 
Los datos disponibles en niños, adolescentes y jóvenes resultan especialmente preocupantes: las tasas de suicidio crecieron del 1.9 al 2.6 por cada 100.000 y del 0.5 al 1.5 por cada 100.000, para las franjas de edad de 10 a 14 en niños, y de 5 a 14 años en niñas, respectivamente. Por exponer unas cifras que ilustren el panorama, en el rango comprendido entre 1980 a 1996 la tasa de suicidio entre personas del rango de edad 15-19 años se incrementó en un 14\%, al tiempo que entre los niños de 10 a 14, lo hizo en un $100 \%$. Actualmente, en Estados Unidos, la tasa de suicidio específica entre los 10 y los 14 años es 1.6 por cada 100.000 , lo que representa el $1 \%$ de todos los suicidios, mientras que en el rango 1519 años es de 9.5 por cada 100.000, representando esta cifra aproximadamente el 6\%. Las chicas hispanas encabezarían esta lista de suicidios. De hecho, en un informe publicado por la Coalicion Nacional de Salud Hispana y Organización de Servicios Humanos (National Coalition of Hispanic Health and Human Services Organizations; COSSHO Report, 1999), un $28 \%$ de las chicas latinas habían considerado seriamente el suicidio, en comparación con el $26 \%$ de chicas afro-americanas y el $22 \%$ de chicas blancas. La investigación de los últimos años ha mostrado que más del $50 \%$ de la población encuestada de edades comprendidas entre los 13 y los 19 años informaron de pensamientos suicidas de carácter intermitente y que del 12 al 15\% habían estado muy próximos a intentarlo (Zayas, Kaplan Turner, Romano y González Ramos, 2000). En la misma línea irían los datos aportados por el Youth Risk Behavior Surveillance, en un informe bianual de los CDC (Centers for Disease Control and Prevention) obtenido mediante sondeos a estudiantes de escuelas públicas y privadas en el que se revela que un 15\% de las adolescentes hispanas de los EEUU ha intentado el suicidio, seguido de un $10.2 \%$ de afroamericanas y un $9.8 \%$ de caucásicas. Al igual que revelan otros estudios, la situación no se limita al intento, sino que también es necesario tener en cuenta los que han considerado esta práctica, es decir, los que tienen cogniciones más o menos recurrentes sobre su ejecución: $25.6 \%$ en el caso de adolescentes hispanas, 22.8\% de caucásicas y 18.7\% de afroamericanas, encontrándose en cualquier caso, con altas tasas de sentimientos depresivos, tales como la apatía, abulia o la tristeza: 46.7\%, 37.9\% y 33.9\% para hispanas, caucásicas y afroamericanas, respectivamente.

Siendo este el estado de la cuestión, no cabe sino preguntarse por el entramado social que subyace a esta problemática, cuyas consecuencias se agudizan al hablar de suicidio en niños y adolescentes como miembros de un medio sociocultural compartido y que, como tal, no deberíamos eludir responsabilidades. Además del mayor impacto y calado social al estar frente a una población más vulnerable y con menos recursos psicológicos y herramientas de afrontamiento ante situaciones traumáticas, en estos casos, se acentúan aún más estas inquietudes acerca de qué variables podrían estar influenciando este tipo de prácticas lo que nos proporcionarían claves orientativas para el diseño y ejecución de programas preventivos.

La confluencia de múltiples factores hace harto difícil determinar con precisión una causa única para consumar este tipo de actos.

Se denomina factor de riesgo a cualquier característica o cualidad de una persona o comunidad que se sabe que va unida a una elevada probabilidad de dañar la salud, es decir, que determinan la vulnerabilidad de un sujeto para cometer actos suicidas, incluyendo lo que se denominan situaciones contextuales, socioculturales y la presencia de psicopatologías específicas (Munist, Santos, Klotiarenco, Suárez, Indante y Grotberg, 1998). 
En el caso de los jóvenes y los adolescentes, la literatura disponible respecto a los factores de riesgo, reincide en subrayar el padecer una enfermedad crónica dolorosa, un trastorno psicológico que no necesariamente ha sido diagnosticado (Dorpat y Ripley, 1960; Barraclough, Bunh, Nelson y Sainsbury, 1974; Arató, Demeter, Rihmer y Somogy 1988; Rich, Ricketts, Fowler y Young, 1988), una tentativa previa de suicidio (Cotton, et al., 1985; Allebeck et al., 1987; Modestin et al., 1992; Taiminen et al., 1994; Rossau et al., 1997; Hawton et al., 2005), la depresión y la esquizofrenia (Nieto y Vieta, 1997) así como, variables concretas de personalidad como un carácter impulsivo con falta de control de las emociones y la alta carga de estrés emocional que padecen. Otros autores recalcan el nivel de desesperanza, la existencia de ideación o plan de suicidio, intentos previos, estado emocional y afectivo, baja densidad en las redes de apoyo social, desintegración social, pérdidas afectivas recientes, estatus mental y deseo de ayuda (Carveth y Glottieb, 1979), siendo señales de alerta un estado de ánimo continuamente deprimido o desesperado; alteraciones en comer y dormir; bajo rendimiento escolar, aislamiento social gradual y una mayor incomunicación con las personas, ruptura en la comunicación con los padres y otras personas significativas en la vida del adolescente, antecedentes de intentos suicidas o complicación en accidentes y comportamiento aparentemente temerario, autodestructivo, poco usual, como el uso frecuente de drogas o alcohol, así como el uso imprudente del automóvil (Cónger, 1980). Durante los últimos años se ha incrementado además el acoso y maltrato por bullying, esto es, el acoso físico o psicológico al que puede verse sometido un alumno por sus compañeros con unas cifras a nivel mundial que apuntan a que lo sufren alrededor de 24 millones de niños y jóvenes al año, bien en forma de acoso o intimidación en el colegio, que sigue siendo el tipo más frecuente, bien en forma de nuevas vertientes como el bullying electrónico o cyberbullying (extendido principalmente en Estados Unidos, Reino Unido, España, Francia, Holanda y Rusia), así como el sexting, o difusión de imágenes o vídeos de alto contenido erótico con el objetivo de humillar a la víctima. Las chicas hispanas se convertirían en una población altamente vulnerable al aunar en muchos casos varios factores de riesgo: embarazos prematuros, intentos previos de suicidios, abuso de drogas y alcohol y posesión de armas. La OMS sitúa también como causas de los suicidios en las regiones de ingresos bajos y medios la presión por problemas socioeconómicos, el haber vivido bajo conflictos bélicos, sufrido abusos, ser víctimas de la violencia, la discriminación...

Las relaciones familiares desempeñan también un papel crucial en el entramado de variables, al ser el primer medio de socialización, sentando las bases de los estilos comunicativos y de personalidad, contribuyendo a la aparición de rasgos que podrían predisponer a la ejecución del acto suicida, entre los que se destaca la propia violencia, la impulsividad, la baja autoestima, las dificultades en las relaciones con personas significativas... En otras ocasiones, pueden darse pautas interactivas donde las familias desempeñen un papel sobreprotector, lo que propiciaría una baja tolerancia a la frustración, a la par que conspira contra el buen desarrollo de la personalidad, lo que desemboca en una gran vulnerabilidad y recursos limitados ante las vicisitudes inherentes a la vida.

Igualmente, se ha apuntado a otras causas que incidirían de una forma menos directa como el clima, la luz, las normas y prácticas culturales, los medios de difusión y los medios interactivos digitales, así como los determinantes sociales, incluidas las decisiones políticas sobre la distribución de recursos y el poder y el ejercicio de los derechos humanos. 
Por el contrario, entre los factores protectores comúnmente señalados que podrían ayudar en la prevención del suicidio destacan el proporcionar un tratamiento efectivo y apropiado para los trastornos (tanto físicos como mentales), establecer redes de apoyo familiares y en la comunidad, dificultar el acceso a métodos letales, así como el entrenamiento en la resolución de problemas.

El presente estudio surge de la necesidad de profundizar en el conocimiento de los factores de riesgo existentes en niños y adolescentes para la consumación de suicidio, en este caso, percibidos a través de terapeutas que trabajan con población en situación de riesgo, lo que nos permitirá un acercamiento a desentrañar mejor las causas que subyacen a esta problemática, información valiosa en el diseño de estrategias de intervención a nivel preventivo.

\section{Método}

\section{Participantes}

La muestra, seleccionada mediante un muestreo intencional, quedó conformada por 593 niños y adolescentes residentes en el sureste de Los Ángeles, (EEUU) más concretamente, procedentes de cuatro centros educativos seleccionados aleatoriamente en las ciudades de La Puente, Santa Fe, Springs y Pico Rivera, todas ellas con un alto número de estudiantes latinos, de edades comprendidas entre los 3 y los 18 años, $(X=12.24 ; D T=3.06)$ (ver tabla 2) pertenecientes a uno de los programas desarrollados por Pacifics Clinics, una asociación sin ánimo de lucro, actualmente la segunda proveedora de salud mental en el estado de Calidornia, concretamente, "Latina Youth Program" (LYP) en el período de tiempo correspondiente a tres cursos académicos, concretamente, los años 2003-204, 2005-2006, y 2008-2009 (132, 259 y 202 participantes, respectivamente). Se trata de jóvenes en situación de riesgo por sus características sociodemográficas o bien por la presencia de algún trastorno psicológico (principalmente de tipo depresivo, ansioso, desórdenes conductuales e incluso déficits de atención), derivados por los educadores escolares, quienes referían haber detectado en ellos, problemas de conducta. En su mayoría estos participantes eran de etnia Latina (89.57\%). La muestra estuvo bastante equilibrada en lo que a género se refiere a género ( $53.2 \%$ chicas y $46.8 \%$ chicos) (ver tabla 3 ).

\section{Instrumentos}

La muestra llegaba referida por los educadores de los centros participantes en el programa, que cumplimentaban una hoja señalando el/ los motivo(s) pertinente(s) entre los que se citan a continuación: irritabilidad, conflicto con los padres, con los compañeros, tristeza, baja autoestima, descenso en la calidad del trabajo escolar, llanto, aislamiento, desesperanza, impulsividad, déficits de atención, pérdidas significativas, posibles desórdenes alimentarios, sueño en clase, síntomas manifiestos de intención de suicidio, abuso de alcohol o drogas, apariencia descuidada..., así como cualquier observación o apunte que consideraban oportuno, y el tiempo aproximado que llevaban percibiendo estos problemas (School-based referral form) (ver anexo 1). 
Tabla 2. Número y porcentaje total de latinos de la muestra analizada durante tres cursos académicos

\begin{tabular}{|c|c|c|c|c|}
\hline Edad & $\mathbf{2 0 0 3 - 0 4}$ & $\mathbf{2 0 0 5 - 0 6}$ & $\mathbf{2 0 0 8 - 0 9}$ & \% Total \\
\hline $\mathbf{3}$ & & 1 & & 0,17 \\
\hline $\mathbf{4}$ & 1 & 3 & & 0,69 \\
\hline $\mathbf{5}$ & 2 & 9 & & 1,89 \\
\hline $\mathbf{6}$ & 3 & 14 & 1 & 3,09 \\
\hline $\mathbf{7}$ & 3 & 15 & 1 & 3,26 \\
\hline $\mathbf{8}$ & 6 & 14 & 8 & 4,80 \\
\hline $\mathbf{9}$ & 8 & 12 & 6 & 4,46 \\
\hline $\mathbf{1 0}$ & 4 & 15 & 2 & 3,60 \\
\hline $\mathbf{1 1}$ & 9 & 21 & 14 & 7,55 \\
\hline $\mathbf{1 2}$ & 19 & 26 & 31 & 13,04 \\
\hline $\mathbf{1 3}$ & 27 & 39 & 48 & 8,35 \\
\hline $\mathbf{1 4}$ & 9 & 19 & 40 & 11,67 \\
\hline $\mathbf{1 5}$ & 16 & 16 & 17 & 8,40 \\
\hline $\mathbf{1 6}$ & 15 & 27 & 7 & 8,40 \\
\hline $\mathbf{1 7}$ & 7 & 13 & 1 & 3,60 \\
\hline $\mathbf{1 8}$ & & 5 & 2 & 1,20 \\
\hline Perdidos & 3 & 10 & 14 & 4,63 \\
\hline TOTAL & 129 & 249 & 178 & 583 \\
\hline
\end{tabular}

Tabla 3. Datos sociodemográficos de la muestra: etnia y sexo

\begin{tabular}{|c|c|c|c|c|}
\hline & \multicolumn{5}{|c|}{$\%$} \\
\hline ETNIA & $\mathbf{2 0 0 3}$ & $\mathbf{2 0 0 5}$ & $\mathbf{2 0 0 8}$ & Total \\
\hline Latina & 92,4 & 84,2 & 92,1 & 89,57 \\
\hline Afroamericana & 2,3 & 2 & 1,5 & 1,93 \\
\hline Caucásica & 5,3 & 9,5 & 4 & 6,27 \\
\hline Otras & & 4,3 & 1 & 2,65 \\
\hline Perdidos & & & 1,5 & \\
\hline SEXO & & & & \\
\hline Mujer & 64,1 & 47 & 48,5 & 53,2 \\
\hline Hombre & 35,9 & 53 & 51,5 & 46,8 \\
\hline
\end{tabular}

En lo relativo a los factores de riesgo, los terapeutas recogían la información mediante entrevistas con los niños y adolescentes, y anotaban la presencia de éstos en una hoja de registro, seleccionando aquellos que estaban presentes entre los 10 que se listaban (los diez factores de riesgo se obtuvieron de la revisión de la literatura existente acerca de la temática) (ver anexo 2). Si era considerado oportuno, se completaba la información con observaciones procedentes de los padres y educadores. En dicha hoja de registro también se anotaba información sociodemográfica como el sexo, la edad, la etnia, el lenguaje preferido para comunicarse en casa o en el centro escolar, una puntuación acerca del Funcionamiento Global (obtenida a través de la Escala de Funcionamiento Global; GAF; DSM-IV-R, 2000) entre otras. 


\section{Procedimiento}

LYP es un programa desarrollado desde 2002 por Pacifics Clinics, una organización sin ánimo de lucro, actualmente la segunda proveedora de Salud Mental en el estado de California, concretamente en la ciudad de Los Ángeles. Tras obtener el consentimiento de los participantes en la participación del programa, se aplicaron diversas entrevistas semiestructuradas de evaluación, con el objetivo de establecer una línea base con la que comparar los resultados tras la intervención. Se obtuvo así información suficiente para completar la hoja de registro anteriormente descrita, con la información pertinente para obtener el estado psicológico y el funcionamiento global, así como los factores de riesgo percibidos, a partir de los cuales orientar las intervenciones. Para el presente estudio se trabajó con los datos de los usuarios del programa de los años 2003-2004, 2005-2006 y 2008-2009.

\section{Resultados}

El análisis de la información aportada por los participantes del estudio permite confirmar la existencia de diez factores principales de riesgo, consistentes con la literatura disponible sobre el tópico, a saber: problemas en la regulación de emociones, pobreza de comunicación en la familia, pobre rendimiento escolar, influencia negativa del grupo de iguales, ideas suicidas, abuso de sustancias, fuga de casa, problemas con la justicia, problemas relacionados con la identidad sexual, así como intentos previos de suicidio.

El factor de riesgo más comúnmente encontrado en la muestra de participantes en el estudio ha sido la regulación de emociones, señalado por un $96.57 \%$ de los encuestados, seguido de la pobreza de comunicación en la familia y de un rendimiento escolar pobre, con un $89.67 \%$ y un $82.73 \%$, respectivamente. Un $41.57 \%$ de los encuestados señaló como factor de riesgo la influencia del grupo de iguales (ver gráfico 1). El resto de factores obtuvieron un porcentaje inferior.

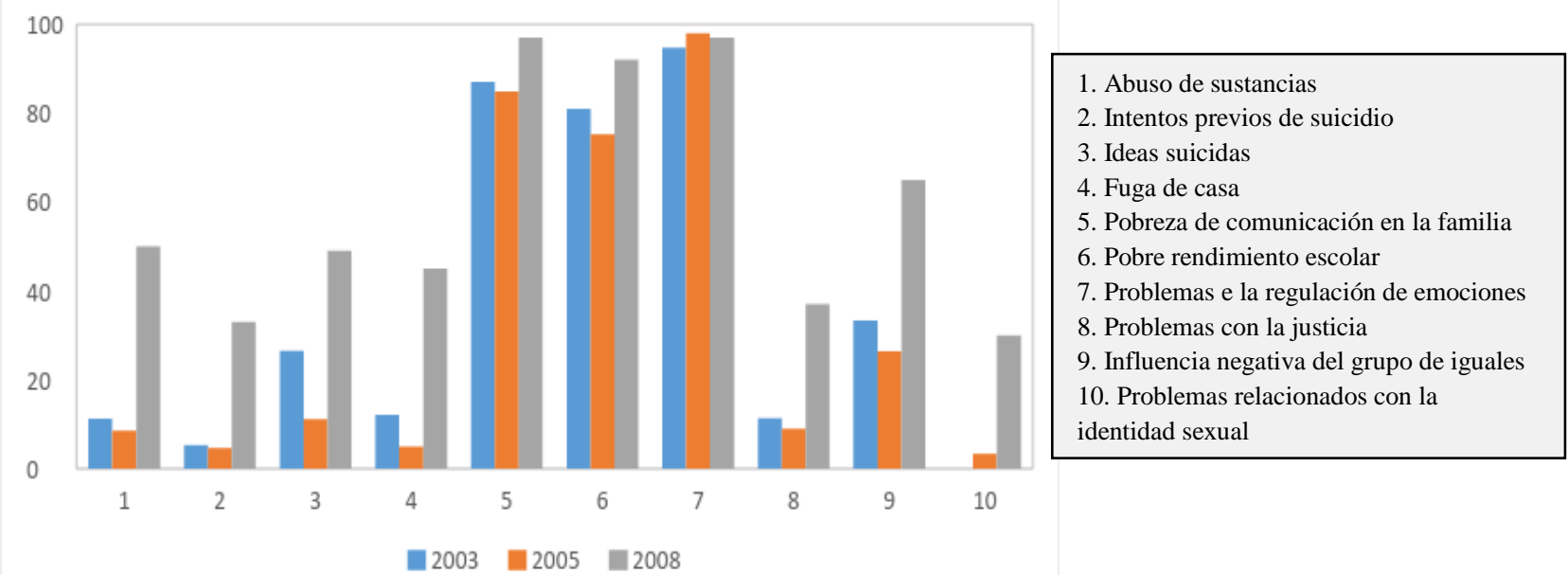

Figura 1. Comparativa en la atribución de factores de riesgo de suicidio entre los años 2003-2004, 2005-2006 y 2008-2009. 
Ahondando en el análisis por año, en 2003-2004 y 2005-2006, los adolescentes coinciden en señalar como principales factores de riesgo problemas en la regulación de emociones, pobreza de comunicación en la familia y pobre rendimiento escolar; en 2008, la pobreza de comunicación en la familia superó en la valoración de riesgo a los problemas en la regulación de emociones y el rendimiento académico pobre. La tendencia encontrada en el último año tenido en cuenta en este estudio (2008-2009), apunta hacia un aumento en la percepción de los restantes factores de riesgo, en comparación con los otros dos años (ver figura 2).
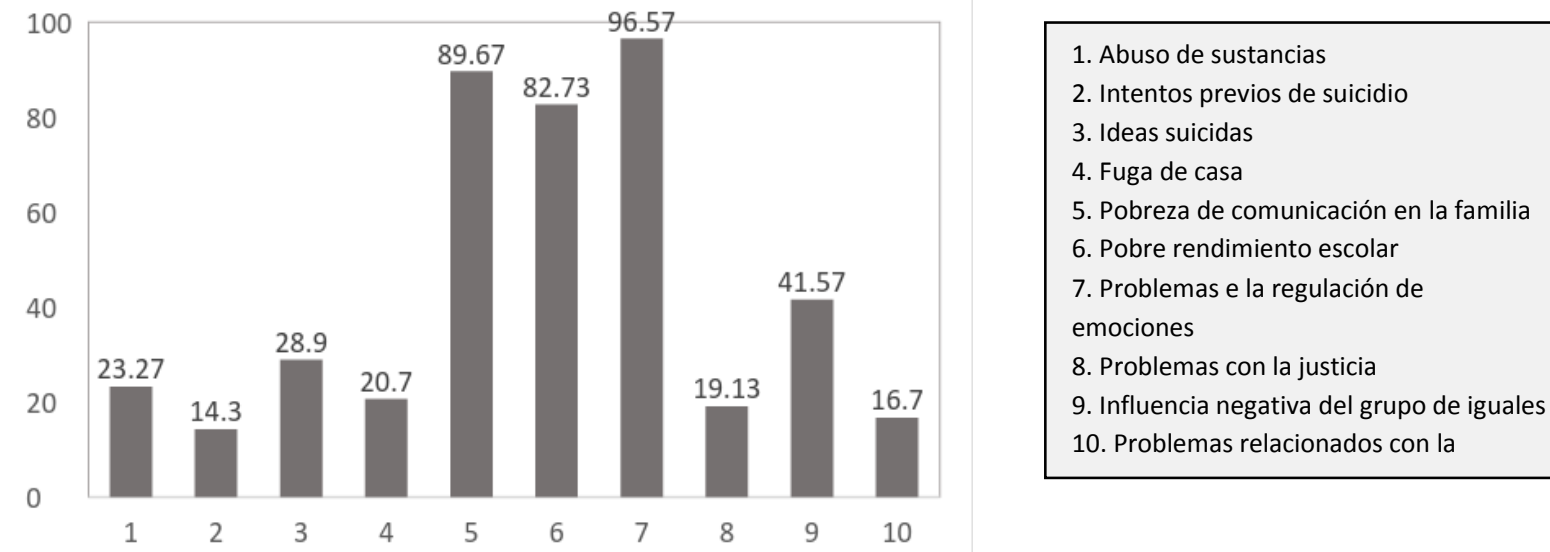

Figura 2. Porcentaje global de sujetos que señalan como factor de riesgo los citados en los cursos escolares 2003-04, 2005-06 у 2008-09.

\section{Discusión}

A pesar del carácter alarmante de las cifras que los últimos estudios nos revelan, el estigma social y el miedo al efecto imitativo de ciertas noticias trágicas, lo que se conoce como efecto Werther, han provocado que, en muchas ocasiones, el hablar de suicidio se haya convertido en un tabú.

Como ya se ha esbozado previamente, y a la luz de los resultados obtenidos del propio autoinforme de la población en riesgo, la cantidad de variables puestas en juego complejizan la determinación precisa de la(s) causa(s) que podrían desembocar en este fatídico desenlace, siendo lo más común un panorama en el que confluyen multitud de variables, un alto número de factores de riesgo, cuyo conocimiento es crucial en el asentamiento de objetivos en la prevención, educación y diseño de estrategias de intervención.

El presente estudio ha permitido confirmar la existencia de diez factores de riesgo comúnmente citados en la literatura, percibidos en la población en riesgo, lo que supone el valor añadido de proporcionar testimonios obtenidos en primera persona, a saber: abuso de sustancias, intentos previos de suicidio, ideas suicidas, fuga de casa, pobreza de comunicación en la familia, pobre rendimiento escolar, problemas en la regulación de emociones, problemas con la justicia, influencia negativa del grupo de iguales, así como problemas relacionados con la identidad sexual.

Los datos anteriores proporcionan claves precisas de enorme utilidad para integrar los componentes necesarios en los programas de detección e intervención. No en vano, la infancia 
y adolescencia pese a ser períodos de especial vulnerabilidad que van a marcar el resto de la vida, son periodos en los que se encuentra una alta sensibilidad a la intervención, ofreciéndose así la oportunidad de corregir la problemática que previamente haya podido emerger, por lo que las intervenciones en estos periodos vitales son altamente recomendables para sentar las bases de una buena salud mental adulta.

Además de los factores de riesgo aquí reportados, y sin obviar el carácter idiosincrásico que envuelve a cada casuística, es preciso hacer un análisis sociocultural del panorama que nos permita un acercamiento genuino a la realidad. Por un lado, algunos adolescentes están particularmente expuestos a padecer problemas de salud y de desarrollo debido a factores derivados del contexto, como podrían ser la marginación, la explotación, o el carecer de apoyo familiar o del grupo de iguales. Por otra parte, el período vital en el que se encuentran, la adolescencia, un período de luchas constantes con el grupo de mayores que los tutelan por la conquista de una mayor autonomía, a la par que aumenta la sensibilidad y vulnerabilidad a las presiones del grupo de iguales, que se convierten en grupo de referencia. Es una afirmación común entre los adolescentes reportar problemas en la gestión y canalización de emociones, de lo que se deriva la ingente cantidad de trabajo que podría realizarse a nivel psicológico, siendo el componente emocional básico en cualquier programa de intervención que se diseñe. Dicho programa, también debería incluir el trabajo con las familias, dada la elevada tasa reportada en lo que a pobreza en la comunicación se refiere. No en vano, la ausencia de calidez familiar, de interacciones favorables comunicativas y un clima de discordia limitan las oportunidades de aprendizaje de resolución de problemas, a la par que privan al adolescente de soporte ante los eventos vitales estresantes. Es conveniente aquí señalar, la importancia que tiene la dinámica familiar en el establecimiento de valores, y que la familia se convierta en un grupo de apoyo, para lo que resulta vital la adopción de pautas comunicativas auténticas y saludables.

Particularizando en el caso de los latinos procedentes de familias inmigrantes, el choque cultural en el que se ven inmersos estos adolescentes también podría desempeñar un nefasto papel: por un lado, la primera generación, los padres, pretenden mantener las costumbres y tradiciones del país de procedencia, el arraigo con su tierra natal, a la par que la segunda generación, que no tiene un apego tan establecido, sumado al deseo de ser acogido dentro del nuevo país, pretenden adaptarse a la nueva cultura, lo que podría provocar conflictos de seriedad diversa dentro del seno familiar, caso de no involucrarse y obstaculizar la elaboración de la identidad bicultural de sus hijos. Una buena comunicación estimula un sentimiento de seguridad, que favorece el crecimiento personal, a la par que la gestión inadecuada de los conflictos en el seno familiar, unida a la ausencia de manifestaciones afectiva en el hogar y a una comunicación escasa o inadecuada se comportan como importantes factores de riesgo para conductas de tipo autodestructivas, forma de comunicación de sentimientos, demandas o súplicas que el adolescente es incapaz de manifestar con estrategias de afrontamiento más saludables.

En esta línea iría también el trabajo en los grupos para padres, así como el trabajo en lo que se denominan esfuerzos comunicacionales (public service announcements), que entre otros objetivos pretenden proporcionar asistencia en el proceso de asimilación de los cambios requeridos por el adolescente en su proceso de individuación, al tiempo que, se trabaja en el contexto familiar para conseguir interacciones de mayor calidad y eficacia, mejorándose la comunicación. La potenciación de la salud de los adolescentes, necesariamente redunda en el 
sector de la educación, a la par que permite reforzar el trabajo familiar en lo que a valores se refiere, además de la consabida labor a nivel académico, siendo un buen período de partida los años de la escuela intermedia, garantizando el acceso a una enseñanza escolar de calidad y a la consecución de objetivos curriculares, a la par que proporcionando educación sanitaria basada en conocimientos prácticos, así como servicios de salud desarrollando campañas de prevención del bullying, tanto en su vertiente tradicional, como cibernética, lo que permitiría maximizar el papel que juega la enseñanza y el contexto académico como factor de protección de influencia significativa. No en vano, el ambiente escolar es un agente protector ante el posible consumo de drogas (Villatoro et al., 1999).

Ante la problemática que se está tratando, se revela imprescindible que los gobiernos establezcan un compromiso respecto del establecimiento y aplicación de un plan de acción coordinado, puesto que actualmente, sólo 28 países cuentan con estrategias de prevención del suicidio y sólo 60 recogen datos fiables sobre las cifras. Quizá motivada por estas cifras, en 1999, la Organización Mundial de la Salud puso en marcha el Programa SUPRE (de las siglas, Suicide Prevention) como una iniciativa a escala mundial para la prevención del suicidio, habiéndose realizado desde entonces diversas publicaciones divulgativas dirigidas específicamente a los sectores implicados en la prevención, tales como los profesionales de la salud, los educadores, los gobiernos, las familias y las comunidades. La Fundación Estadounidense para la Prevención del Suicidio (American Foundation for Suicide Prevention, AFSP) por su parte, avisa de la necesidad de atender a las señales de alerta, informando de que los programas de prevención de suicidio con más probabilidades de éxito son los orientados a la identificación y tratamiento de trastornos psicológicos, del efecto del estrés y de los comportamientos agresivos.

Entre las estrategias concretas de intervención, aquellas que contemplan la restricción del acceso a métodos comunes de suicidio, como las armas o sustancias tóxicas, o el adecuado tratamiento en la depresión y abuso de alcohol y sustancias, y el adecuado seguimiento de aquellos que tienen antecedentes de tentativas, han mostrado ser eficaces. En cualquier caso, es indiscutible la necesidad de diseñar programas y estrategias de intervención que necesariamente se deriven de la información proporcionada por la investigación epidemiológica y estadística, poniéndose de relieve el binomio práctica-teoría, permitiendo esta última inferir patrones del estado de salud psicológica de una población, demandando una mayor implicación de la investigación científica, la educación en las familias, escuelas y centros de atención médica, a la par que realzando el papel de la prevención. que mitiguen este acuciante lastre.

\section{Referencias}

Allebeck, P., Varla, A., Kristjansson, E. y Wistedt, B. (1987). Risk factors for suicide among patients with schizophrenia. Acta Psychiatrica Scandinavica, 76, 414-419.

American Psychiatric Association. (2000). DSM-IV-TR. Manual diagnóstico y estadístico de los trastornos mentales. Texto revisado. Barcelona: Masson.

Arató, M., Demeter, E., Rihmer, Z. y Somogy, E. (1988). Retrospective psychiatric assessment of 200 suicides in Budapest. Acta Psychiatrica Scandinavica, 77, 454-456. 
Barraclough, B., Bunh, J., Nelson, B. y Sainsbury, P. (1974). A hundred cases of suicide: clinical aspects. British Journal of Psychiatry, 125, 355-373.

Carveth, W. B. y Gottlieb, B. H. (1979). The measurement of social support and its relation to stress. Canadian Journal of Behavioral Sciences, 11, 179-188.

Conger, J. (1980). Adolescencia. Generación presionada. México: Harla.

Cotton, P.C., Drake, R.E. y Gates, C. (1985). Critical treatment issues in suicide among schizophrenics. Hospital \& Community Psychiatry, 36, 534-536.

Dorpat, T.L. y Ripley, H.S. (1960). A study of suicide in the Seattle area. Comprehensive Psychiatry, 1, 349-359.

Hawton, K., Sutton, L., Haw, C., Sinclair J. y Deeks, J.J. (2005). Schizophrenia and suicide: systematic review of risk factors. British Journal of Psychiatry, 187, 9-20.

Jiaquan X., Shery L. M., Kenneth D. K y Brigham A. B. (2016). Deaths: Final Data for 2013. National Vital Statistics Reports 64, 2.

Modestinm, J., Zarro, I. y Waldvogel, D. (1992). A study of suicide in schizophrenic inpatients. British Journal of Psychiatry,160, 398-401.

Munist, M., Santos, H., Klotiarenco, M.A., Suárez, E., Indante, F. y Grotberg, E. (1998). Manual de identificación y promoción de la resiliencia en niños y adolescentes. OPSOMS-Fundación W: K Kellogg-Fundación sueca para el desarrollo internacional (ASDI).

Nieto, E. y Vieta, E. (1997). La conducta suicida en los trastornos bipolares. En: Trastornos bipolares, E. Vieta y C. Gastó (eds.), pp.318-336. Barcelona: SpringerVerlag Ibérica:

Organización para la Cooperación y el Desarrollo Económicos. (2014). Suicides, OECD Factbook 2014: Economic, Environmental and Social Statistics. Paris: OECD Publishing.

Organización Mundial de la Salud. (1999). The world health report 1999-making a difference. Ginebra: Organización Mundial de la Salud.

Organización Mundial de la Salud. (2004). Suicidio, un problema de salud pública enorme y sin embargo prevenible. Ginebra: Organización Mundial de la Salud.

Organización Mundial de la Salud. (2006). Prevención del suicidio. Recursos para consejeros. Ginebra: Organización Mundial de la Salud.

Organización Mundial de la Salud. (2014). Prevención del suicidio. Un imperativo global. Ginebra: Organización Mundial de la Salud.

Rich, C.I., Ricketts, J.E., Fowler, R.C. y Young, D. (1988). Some differences between men and women who commit suicide. American Journal of Psychiatry, 145,718-722.

Rossau, C. y Mortensen, P. (1997). Risk factors for suicide in patients with schizophrenia: nested case-control study. British Journal of Psychiatry, 171, 355-359.

Taiminen, T.J. y Kujari, H. (1994). Antipsychotic medication and suicide risk among schizophrenic and paranoid in-patients. Acta Psychiatrica Scandinavica, 90, 247-251.

Villatoro, J., Medina-Mora, M.E., Cardiel, H., Villa, G., Alcántar, E., Vázquez, L., Fleiz, C., Navarro, C., Blanco, J. y Néquiz, G. (1999). Consumo de drogas, alcohol y tabaco en estudiantes del Distrito Federal: medición otoño 1997. Reporte Global de Escuelas Secundarias, SEP, IMP, México.

Zayas, L., Kaplan, C., Turner, S., Romano, K. y Gonzalez-Ramos, G. (2000). Understanding Suicide Attempts by Adolescent Hispanic Females._Social Work, 45, 53-62. 
ANEXO 1. School-based referral form

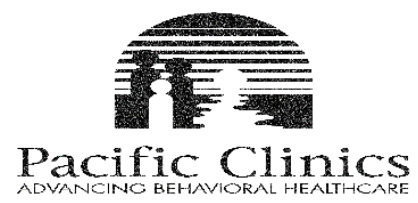

Person making referral

Last Name

First Name

Position

Classroom Ext.

Student being referred

Last Name,

Age

Grade

Legal Guardian

Last Name,

First Name

Relationship

Address

\section{Phone (cell/home)}

Date Caregiver/Parent and Student were notified of this referral:

Home Language

Does this student have Medi-cal?: Yes No

Is this student uninsured (no insurance at all)? Yes No

Reason(s) for Referral (check all that apply)

Anger/Irritability Conflict with parents Conflict with peers Sadness Low self-esteem Decrease in quality of school work Attendance Problem__ Grief/Loss issues Tearfulness Isolation/withdrawn Possible Eating Disorder Hopelessness Falling asleep in Overall appearance is disheveled/unkempt

\section{Insurance Verification}

Medi-Cal

\#

or

Social Security
class__Suicidal statements/gestures___Drug/alcohol use/abuse

Is there any further information that you feel is pertinent to this student's referral?

How long have you observed the above behaviors?

I, the Parent/Legal Guardian of the above named student, hereby authorize the exchange of information pertinent to the student and this referral between the school/school district and Pacific Clinics.
Parent/Legal Guardian
Date

For Office Use Only: Date Received by Supervisor and Initials

Date Received by Clinician: \& Printed Name 
ANEXO 2. Hoja de registro factores de riesgo

ECHO I.D. MINI CHART:

REV0512

SEX: $\_$F _

GRADE:

ETHNICITY : _ L L C C _ AA _

INSURANCE: EPSDT UNKNOWN NONE PRIVATE

SCHOOL NAME:

SCOOL LOCATION (CITY):

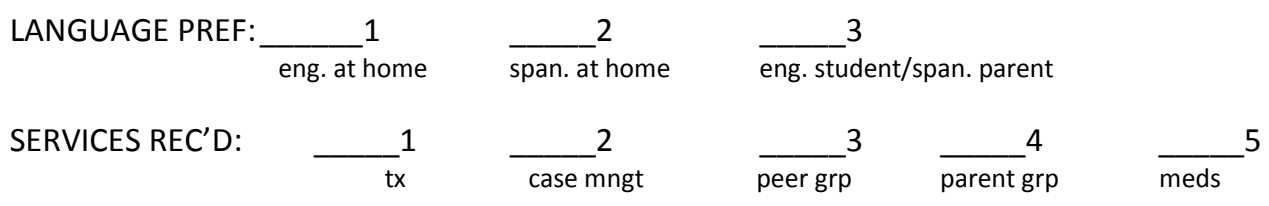

PLACE SERVICES REC'D: school home prog. Office other EBP: __ Truma Focused CBT _ _ Functional Fam. Tx. __ Interpersonal Tx. Seeking Safety __ ART __ Managing \& Adapting Practices Other (list) MONTHS IN TX: SEXUAL IDENTITY ISSUES: YES NO

RISK FACTORS: PROGRESS ON RISK FACTORS:

(rate factors on a scale from $1-10$, where 1 is very mild and 10 is very severe)

\begin{tabular}{|c|c|c|}
\hline (at intake) & & (present/close) \\
\hline 1 & substance use/abuse & 1 \\
\hline 2 & suicide ideation & 2 \\
\hline 3 & suicide attempt & 3 \\
\hline-4 & run away behavior & -4 \\
\hline 5 & communication prob & -5 \\
\hline 6 & poor schl. funct. & -6 \\
\hline 7 & diff. reg. emotions & -7 \\
\hline 8 & legal/juvenile sys & -8 \\
\hline -9 & neg. peer influence & 9 \\
\hline 10 & sexual identity & 10 \\
\hline
\end{tabular}

DIAGNOSITIC CODE(S) intake: DIAGNOSTIC CODES(S) present/close: GAF: $\mathrm{X} 1$ $\mathrm{X} 2$ 